\title{
Semi-empirical dynamic photospheric models of bright solar flare
}

\author{
K. V. Alikaeva ${ }^{1} \dagger$ and N. N. Kondrashova ${ }^{1}$ \\ ${ }^{1}$ Main Astronomical Observatory, National Academy of Sciences of Ukraine, \\ email: alikaeva@mao.kiev.ua, kondr@mao.kiev.ua
}

\begin{abstract}
The Fraunhofer spectra were used to study the physical condition variations in the photospheric layers in the course of a bright solar flare. The observations involve both the impulsive and main phases of the flare. The spectral lines are formed in the photosphere at the interval $12-500 \mathrm{~km}$. The modelling was made by fitting the observed and calculated line profiles using the SIR program. The models represent the distributions of temperature, gas density, line-of-sight velocities, etc. on the height. They show some inhomogeneities of these physical parameters at various photospheric levels. Their significant deviations from the VAL-F model may be seen in the course of the flare. The largest heating of the high and middle photospheric levels take place close to two X-ray maxima. At the final flare stage the photosphere is disturbed appreciably while the spectral data testify to the flaring process fading in the chromosphere. This may be an evidence of a disturbance propagation from the upper atmospheric levels to the low ones in the impulsive and main phases.
\end{abstract}

The flare on October 7, 1979 occurred at 07:55 UT and lasted for at least an hour. The X-ray flux (0.05-0.4 nm) fluctuations show spikes with maxima at 08:00 UT and 08:09 UT. A series of Fraunhofer spectra were acquired with the solar horizontal telescope of the Main Astronomical Observatory (Kyiv, Ukraine). The observational period involves both the impulsive and main phases of the flare. The Fraunhofer lines used for modelling are formed in the height interval from 12 to $500 \mathrm{~km}$. The modelling was made by fitting the observed and calculated line profiles by use the SIR (Stokes Inversion)-program (Ruiz Cobo \& Toro Iniesta (1992)).

The initial two moments of the spectral observations coincide with the first low X-ray spike at 08:00 UT. The temperature and gas pressure distributions reveal a considerable temperature increasing in the high photosphere (see figure 1). The heating propagates up to the height of $170 \mathrm{~km}$. During $30 \mathrm{~s}$ the temperature excess (relative to bright plage VAL-F model (Vernazza et al. (1981))) increased from $1700 \mathrm{~K}$ (at the first observation moment) up to $4000 \mathrm{~K}$. The heating of the high photosphere is accompanied by gas pressure decreasing up to 2 orders at the second moment.

At the flare final stage the photosphere much yet remains to be disturbed (see figure 2) while the $H_{\alpha}$-line intensity differs slightly from that in the undisturbed solar atmosphere. This fact is an evidence of the disturbance propagation from the upper atmosphere to the photosphere during the flare.

The main properties of models obtained are as follows:

-The inhomogeneous structure of flaring region at the various photospheric levels is a general property of the calculated models.

-The heating of the high and middle flare photosphere takes place during the whole time interval of the observation.

$\dagger$ Present address: Main Astron. Observ., 27 Akademika Zabolotnoho St., 03680 Kyiv, Ukraine. 


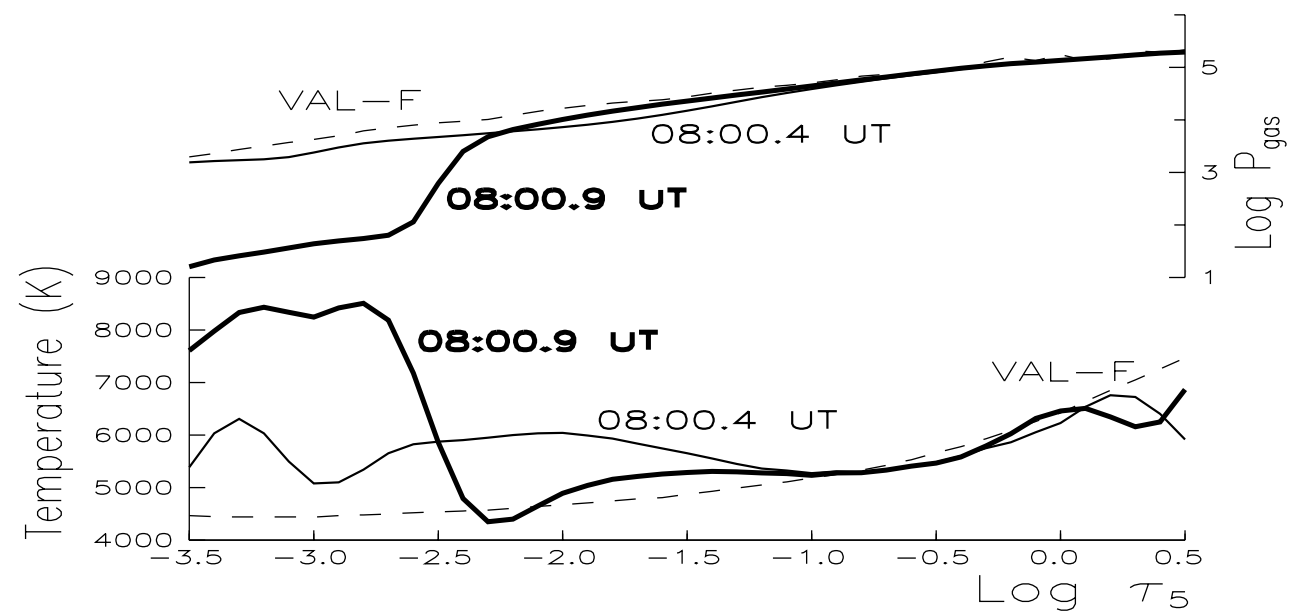

Figure 1. The temperature and gas pressure distributions at the first X-ray spike maximum.

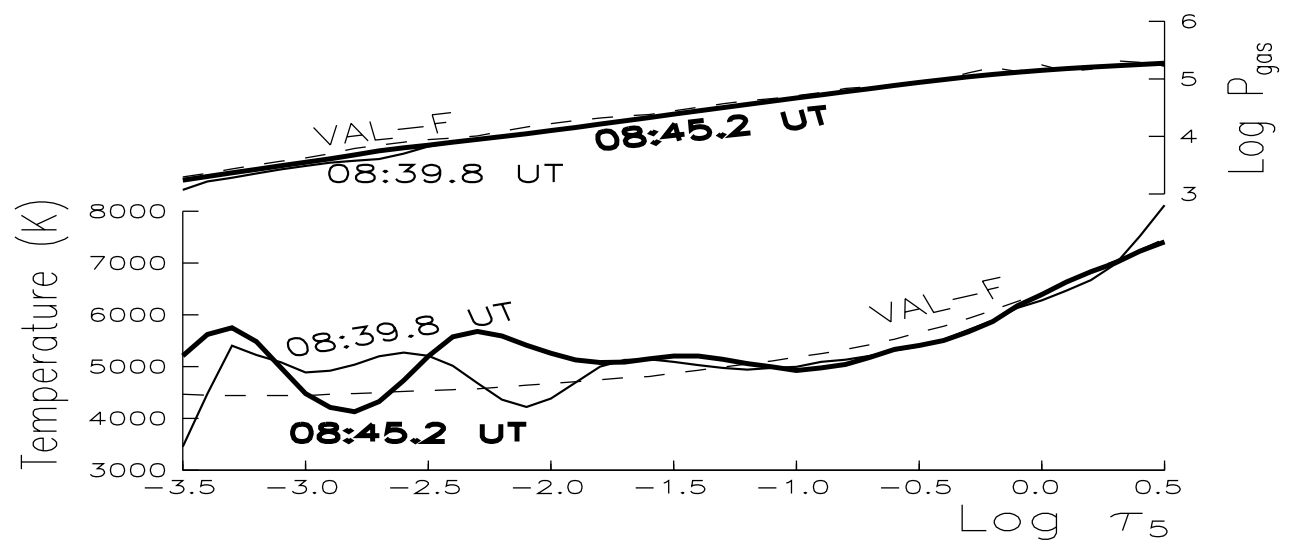

Figure 2. The same as in Figure 1 for the final stage of the flare.

-All the models show the slight temperature deficit at the lower photosphere. The $T$ inversion is found at the $160-200 \mathrm{~km}$ level.

-The gas pressure deficit is revealed, particularly, at the upper photospheric levels.

- The layers of ascending matter alternate with the layers of descending matter.

-The large-scale motions are suppressed during the flare, mainly, before the impulsive phase. A tendency to $V_{m a}$ increase in the course of the flare evolution is revealed.

Our results indicate that the flare photosphere is an equivalent component of the flare processes. It is need to take into account when the theories of flares are created.

\section{References}

Ruiz Cobo, B. \& Toro Iniesta, J.C., 1992, ApJ, 398, 375.

Vernazza, J.E. et al., 1981, ApJ Suppl., 45, 635. 